\title{
Multi-band Carrier-less Amplitude and Phase Modulation for VLC: An Overview
}

\author{
P. Chvojka, K. Werfli, P. A. Haigh, S. Zvanovec, Z. Ghassemlooy and M. R. Bhatnagar
}

\begin{abstract}
The rapid development of solid-state lighting technologies has been the stimulus for visible light communications (VLC) to be the focus of enormous interest over the last decade. The key feature of simultaneous data transmission and illumination using white light-emitting diodes (LEDs) makes VLC a potential candidate for future power efficient communication networks that aim to meet the ever-increasing demands for high-speed internet services. Researchers, motivated by the success of VLC technology, have developed a number of techniques and methods to support communication systems with both high transmission speeds and spectral efficiency. Here, we provide an overview of the multi-band carrier-less amplitude and phase ( $m$-CAP) modulation technique enabling highly spectrally efficient VLC links in bandlimited environments.
\end{abstract}

Index Terms - multi-band carrier-less amplitude and phase modulation, spectral efficiency, visible light communications

\section{INTRODUCTION}

$\mathrm{T}$ HE existing radio frequency (RF) based wireless communication technologies cannot cope with the increasing demands for high-speed data traffic, which is expected to grow sevenfold between 2016 and 2017 [1]. The commercially available RF spectrum has already been exhausted, thus leading to the spectrum crunch that needs urgent attention. There are a number of possible options to address this problem including allocation of new spectra, which is highly expensive, spectrum hoping, sharing and borrowing, advanced modulation and coding schemes, interference elimination, and parallel transmission. Alternatively, the carrier frequency could be shifted to the optical domain i.e., optical wireless communications (OWC), which covers the three main bands of visible, infrared and ultraviolet, thus offering an alternative solution for future broadband services in indoor and outdoor environments [2]. The infrared and ultraviolet bands are mostly used in outdoor applications for line-of-sight (LOS) and non-LOS links, whereas the visible band has been widely adopted in the indoor environment [3]. As a part of OWC, visible light communications (VLC) has been the focus of enormous attention due to a few key advantages including (i) high and unregulated bandwidth (in the order of $\mathrm{THz}$ );

P. Chvojka and S. Zvanovec are with the Department of Electromagnetic Field, Faculty of Electrical Engineering, Czech Technical University in Prague, Technicka 2, Prague 16627, Czech Republic (e-mail: chvojpe8@fel.cvut.cz; xzvanove@fel.cvut.cz).

K. Werfli and Z. Ghassemlooy* are with the Optical Communications Research Group, Northumbria University, Newcastle upon Tyne NE1 8ST, UK. (ii) immunity to electromagnetic interference; and (iii) inherent security, as light is confined within walls. VLC systems benefit from current solid state lighting (SSL) technologies, utilizing white light-emitting diodes (LEDs), which are already widely used in buildings etc. Employing LEDs bring additional benefits such as cost-effectiveness and low power consumption. Thus, VLC could be seen as an ideal possible candidate in multi-technology fifth generation (5G) wireless access networks, which are expected to be deployed beyond 2020 [4]. Therefore, VLC based wireless systems utilizing existing lighting infrastructures are a promising and complementary way forward to meeting the aforementioned bandwidth demand. In addition, VLC systems could readily be deployed in an Internet of Things (IoT) context within wider smart networks $[5,6]$. Despite a number of benefits, there are several challenges in VLC that require research, namely a low LED modulation bandwidth (in the order of $\mathrm{MHz}$ ), dimming control or shadowing of the LOS communications link.

To achieve higher transmission speeds in bandlimited VLC systems, a number of techniques have been reported [7-9]. Spectrally efficient modulation formats such as orthogonal frequency division multiplexing (OFDM), pulse amplitude modulation (PAM) or carrier-less amplitude and phase (CAP) modulation are popular options for increasing data rates. Multilevel PAM ( $L$-PAM) is an attractive scheme due to its high spectral efficiency (increased by the higher number of levels), but at the cost of lower power efficiency. However, L-PAM systems are sensitive to the nonlinear electro-optics characteristics of the LEDs. To overcome this, in [10] a superposed PAM system utilizing spatially separated LEDs was demonstrated to transmit signals with different amplitudes. The signals combined by superposition in free space results in multi-level modulation. OFDM is very popular in bandlimited VLC systems due to the relatively simple implementation using inverse fast Fourier transforms (IFFTs) and FFTs, higher data rates and robustness to inter-symbol interference (ISI) [11, 12]. However, power efficiency in OFDM needs to be addressed due to the high peak-to-average power ratio (PAPR), which ultimately results in signal distortion due to nonlinearity of the LED sources $[13,14]$.

* Is with QIEM, Chinese Academy of Sciences, Fujian, China (e-mail:khald.werfli@northumbria.ac.uk; z.ghassemlooy@northumbria.ac.uk). P. A. Haigh is with the Department of Electronic and Electrical Engineering, University College London, WC1E 6BT, UK (e-mail: p.haigh@ucl.ac.uk)

M. R. Bhatnagar is with the Department of Electrical Engineering, Indian Institute of Technology Delhi, New Delhi, 110016, India (e-mail: manav@ee.iitd.ac.in). 


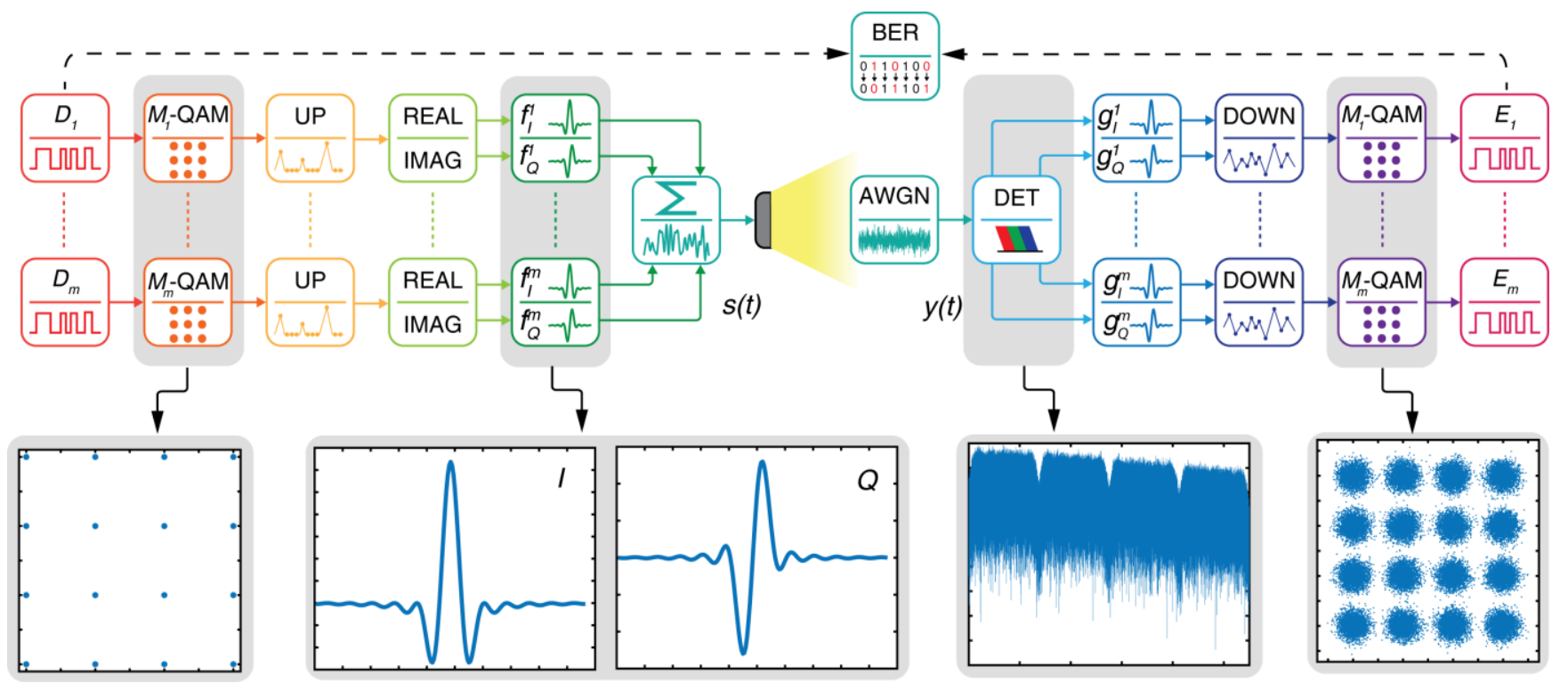

Fig. 1: The $m$-CAP schematic block diagram. The data streams $D_{m}$ are generated and mapped into the appropriate QAM constellation. The signal is upsampled ('UP'), split into real and imaginary parts and passed through the pulse shaping transmit filters. The output signal $s(t)$ is generated as a sum of $m$ individual signals and used to modulate an LED. After passing the AWGN channel, the signal is detected ('DET') and matched filtering is performed. Downsampling ('DOWN') and demodulation follows before the received bits are estimated ('E') and BER is calculated. Inset are examples of 16-QAM constellation diagram at the Tx, the impulse responses of $I$ and $Q$ pulse shaping filters for the subcarriers $n_{l}$, the frequency spectrum and constellation diagram of the received signal.

The experimental work reported in [15] showed that CAP modulation outperforms OFDM in terms of transmission speed using the same physical link. Nevertheless, a flat frequency response, which is required for optimal CAP performance, represents a substantial challenge towards implementing CAP in VLC. Thus, a multi-carrier CAP ( $m$-CAP) scheme was introduced (at first for optical fiber links) by splitting the available bandwidth into $m$ sub-bands (sub-carriers) $[16,17]$. This relaxes the flat-band response requirement and allows utilization of bit- and power-loading algorithms in order to adapt a number of bits/symbol to every individual sub-carrier.

In this paper, we give an overview of the $m$-CAP format adopted in VLC systems. First, the principal of $m$-CAP is described in Section II. Both experimental and theoretical research works are then discussed in Section III. Finally, conclusions and our vision of the future of $m$-CAP are given in Section IV.

\section{PRINCIPLE OF M-CAP}

The schematic block diagram of an $m$-CAP system is illustrated in Fig. 1. First, $m$ independent data streams $D_{n}$, where $n=\{1,2, \ldots, m\}$, are generated and mapped into $M$-quadrature amplitude modulation (QAM) symbols, where $M=2^{k}$ represents the order of QAM, and $k$ represents the number of bits/symbol. The up-sampling ('UP' in Fig. 1) by means of zero padding follows and data is split into the real (in-phase $I$ ) and imaginary (quadrature $Q$ ) components. The $I$ and $Q$ data are then passed through $I$ and $Q$ square-root raised cosine (SRRC) pulse shaping filters, respectively. Note that, $2 m$ filters are utilized in both the transmitter (Tx) and the receiver (Rx), thus resulting in an overall $4 m$ filter requirement in the proposed $m$-CAP system, which introduces a major computational complexity issue. The transmit filters are finite impulse response (FIR) filters with the impulse responses orthogonal in the time domain i.e., $90^{\circ}$ phase shifted, resulting in the formation of a Hilbert pair. They are generated as a product of a cosine and sine waves, with the impulse response of the SRRC filter for $I$ and $Q$ filter, respectively as shown in Fig. 1 for the $1^{\text {st }}$ sub-carrier $n_{1}$, respectively. The carrier frequencies of the individual sub-carriers are given by the frequency of a cosine/sine wave. The impulse responses for $I$ and $Q$ filters are given by $[18,19]$ :

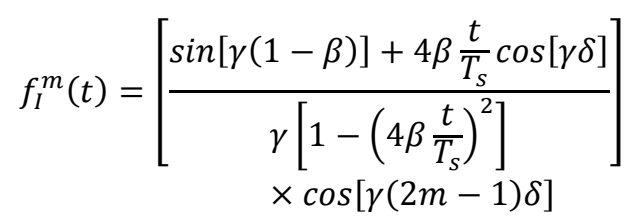

and

$$
f_{Q}^{m}(t)=\left[\begin{array}{c}
\frac{\sin [\gamma(1-\beta)]+4 \beta \frac{t}{T_{s}} \cos [\gamma \delta]}{\gamma\left[1-\left(4 \beta \frac{t}{T_{s}}\right)^{2}\right]} \\
\times \sin [\gamma(2 m-1) \delta],
\end{array}\right]
$$

respectively, where $T_{s}$ is the symbol duration, $\gamma=\pi t / T_{s}$ and $\delta=1+\beta$. The SRRC filter roll-off factor $\beta$ is set within the range of $0 \leq \beta \leq 1$, which determines the bandwidth requirement. Hence, the total signal bandwidth is then given as $B_{\text {tot }}=B_{\text {sig }}(1+\beta)$, where $B_{\text {sig }}$ is the signal bandwidth (or the symbol rate). Note that, lower values of $\beta$ are typically used to increase spectral efficiency as far as possible, as can be found in the literature [16]. The output signal of the $m$-CAP Tx, which is used for intensity modulation of an LED, is given by [18]: 


$$
s(t)=\sqrt{2} \sum_{n=1}^{m}\left(s_{I}^{n}(t) \otimes f_{I}^{n}(t)-s_{Q}^{n}(t) \otimes f_{Q}^{n}(t)\right),
$$

where $\otimes$ represents the time domain convolution and $s_{I}^{n}(t)$ and $s_{Q}^{n}(t)$ are $I$ and $Q M$-QAM symbols for the $n^{\text {th }}$ subcarrier, respectively. The received signal is given as:

$$
y(t)=\Re R s(t) \otimes h(t)+n(t),
$$

where $h(t)$ is the channel impulse response, $\Re$ and $G$ stand for the responsivity and the gain of the photodiode, respectively, $n(t)$ is the additive white Gaussian noise (AWGN) with zero mean and variance of $N_{0} / 2$, where $N_{0}$ is the power spectral density.

Following detection at the $\mathrm{Rx}$, matched filtering is performed using $I$ and $Q \mathrm{Rx}$ filters $g_{I}^{m}(t)=f_{I}^{m}(-t)$ and $g_{Q}^{m}(t)=f_{Q}^{m}(-t)$, respectively. The data is then downsampled ('DOWN' in Fig. 1) and de-mapped into the $M$-QAM symbols. The received bit sequences are then estimated (' $E$ ' in Fig. 1) to determine the link bit error rate (BER). Further details on $m$-CAP modulation can be found in $[20,21]$ and the references within.

\section{Performance AnAlysis of M-CAP systems}

As already stated, the $m$-CAP scheme was originally designed for fiber communication systems, which resulted in the slightly increased data rate, higher bandwidth efficiency and lower dispersion compared to a traditional 1-CAP [16]. In [17], the multi-band approach was reported to support multi-user access network with $9.3 \mathrm{~Gb} / \mathrm{s} /$ user. A number of research reports can be found in the recent literature illustrating the interest and feasibility of $m$-CAP for fiber infrastructures [22, 23]. A novel three-dimensional (3D) $m$-CAP system was investigated in [24] showing the improved performance in the fiber dispersion over the conventional 3D-CAP scheme. Thus, motivated by the aforementioned results, the $m$-CAP scheme was adopted for VLC applications.

\section{A. Theoretical Studies}

Detailed analysis of the $m$-CAP performance in a bandlimited VLC system was reported in [20]. The BER performance of the individual sub-carriers were determined using realistic system and device models through numerical simulations. It was shown that higher order sub-carriers are more prone to the attenuation introduced by the LED frequency response, which significantly degradates their BER performance as illustrated in Fig. 2 for a 5-CAP system. Moreover, it was found that the predicted spectral efficiency can approach $\sim 10 \mathrm{~b} / \mathrm{s} / \mathrm{Hz}$ for $m=20$, which is promising for future spectrally efficient optical systems.

Another theoretical study of $m$-CAP was reported in [19] showing an improvement in the data rate by up to $40 \%$ compared to the traditional 1-CAP in a highly bandlimited system (i.e., the LED modulation bandwidth, which was $1 / 10$ of the available $B_{s i g}$ ). Next, in [25] $m$-CAP with a decision

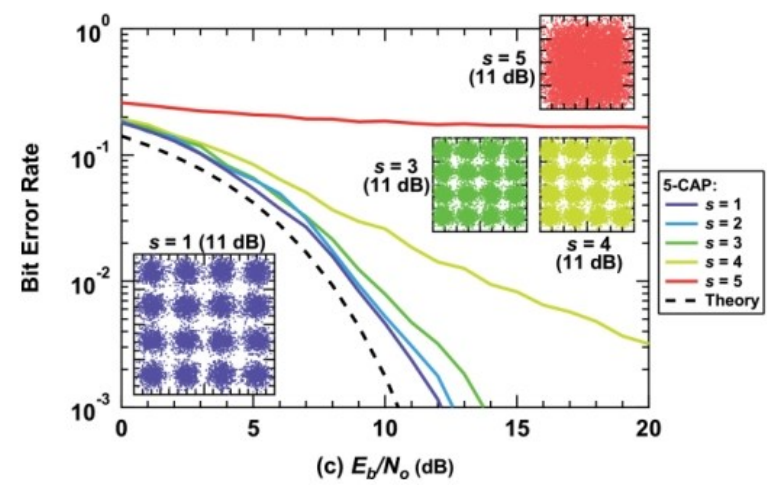

Fig. 2: The BER against the SNR for 5-CAP scheme showing the BER improvement for low order sub-carriers [20].

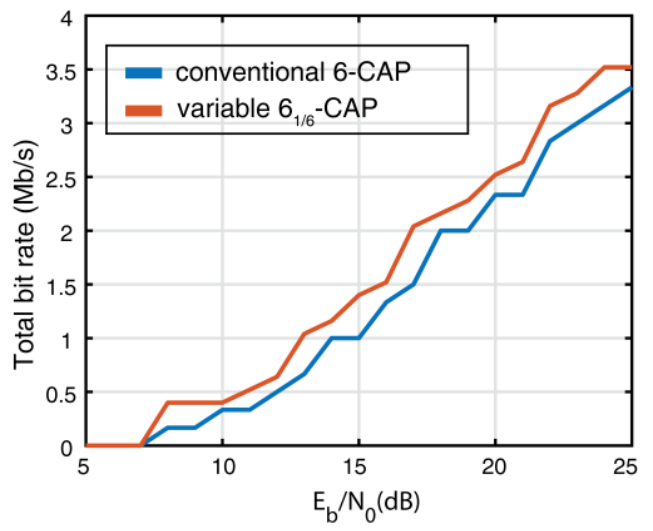

Fig. 3: The comparison of the predicted total bit rates against the bit energy to noise ratio $\left(E_{b} / N_{0}\right)$ for conventional and variable 6-CAP schemes [27].

feedback equalizer (DFE) was investigated showing an enhanced BER performance for lower order $m$-CAP compared to higher order systems where DFE fails to introduce BER improvement.

Normally, the sub-carriers are distributed evenly within the $B_{t o t}$ in $m$-CAP. However, the unequal sub-carrier distribution introduces additional performance improvements as demonstrated in $[26,27]$. In comparison to conventional $m$-CAP, allocating the entire LED modulation bandwidth to the $1^{\text {st }}$ sub-carrier while the rest of sub-carriers are split equally over the remaining $B_{t o t}$ (i.e., outside the LED modulation bandwidth) results in: (i) decreased computational complexity by reducing the number of FIR filters by $80 \%, 75 \%, 67 \%$ and $50 \%$ for $m=10,8,6$, and 4 , respectively [26], which is significant; and (ii) improved achievable data rates by up to $36 \%$ for $m=6$, see Fig. 3 [27].

The Tx and Rx FIR filter parameters have a substantial impact on the system performance and computational complexity. In [21], the BER performance of the m-CAP system using different filter parameters (i.e., $\beta$ and the filter length $L_{s}$ ) was investigated using numerical simulations. It was demonstrated that, both filter parameters considerably improve the system BER performance, see Figs. 4 (a) and (b) for a 5-CAP link. Deploying filters with $L_{s}>12$ was shown to be impractical, which is an important finding towards real time implementation. Both parameters were demonstrated to significantly impact channels with higher signal-to-noise ratio (SNR) levels. 
$E_{b} / N_{0}=10 \mathrm{~dB} \bigcirc E_{b} / N_{0}=20 \mathrm{~dB} \underline{\underline{n=1}} \underline{\underline{n=2}} \underline{\underline{n=3}} \underline{\underline{n=4}} \underline{\underline{n=5}}$

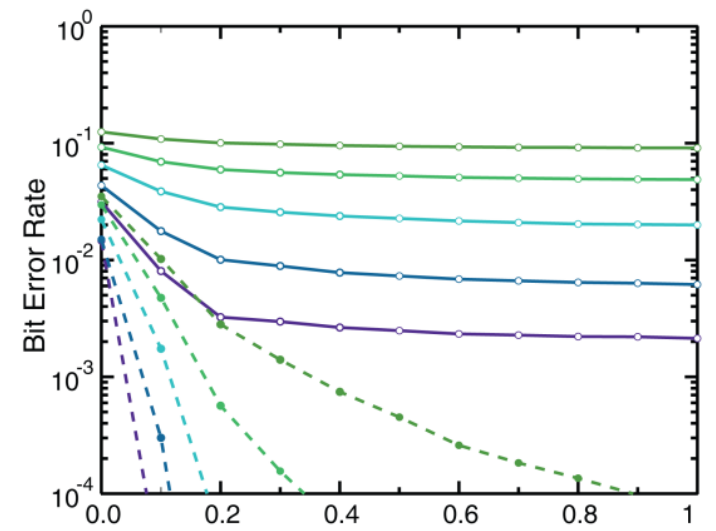

(a) Roll-off Factor $\beta$

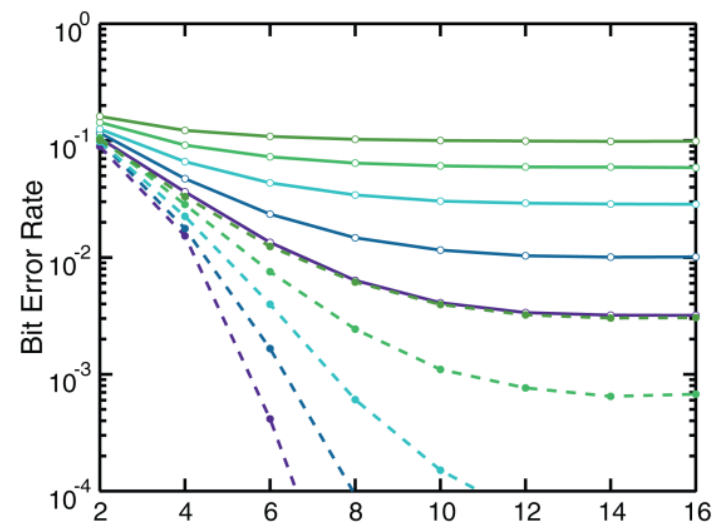

(b) Filter Length (symbols)

Fig. 4: The BER performance of 5-CAP system against: (a) the roll-off factor $\beta$, and (b) filter length $L_{s}$. Increasing both parameters improve the BER performance significantly [21].

\section{B. Experimental Demonstrations}

To date, the number of experimental reports of the $m$-CAP format in VLC is limited, since the majority of prior art focused mainly on the theoretical investigations as discussed above. In [28], a high-speed VLC system based on 3-CAP 64-QAM for a multi-user scheme supporting an overall bit rate of $1.35 \mathrm{~Gb} / \mathrm{s}$ for a BER of $3.8 \times 10^{-3}$ was demonstrated. The individual sub-carriers were transmitted on different wavelengths of the individual chips of the red-green-blue (RGB) LED. On the other hand, it was shown that the multiband approach has higher requirements on PAPR compared to 1-CAP, which is a substantial drawback.

The concept of $m$-CAP was further demonstrated in $[18,29]$ by increasing the order of $m$-CAP. In [18], the available bandwidth was split into $m=\{2,4,6,8,10\}$ sub-carriers and the measured results were compared to the conventional 1-CAP system, see Fig. 5. To maximize system performance, a bit loading algorithm was applied. As can be seen, the higher throughput is supported when increasing the system order $m$. For the 10-CAP scheme, the bit rate and spectral efficiency are $31.53 \mathrm{Mb} / \mathrm{s}$ and $4.85 \mathrm{~b} / \mathrm{s} / \mathrm{Hz}$, respectively, with the BER below the $7 \%$ forward error correction (FEC) limit $3.8 \times 10^{-3}$. At the time of writing, it was the highest spectral efficiency in VLC measured at a transmission distance of $1 \mathrm{~m}$. Thus, the report demonstrated that a higher number of sub-carriers results in a

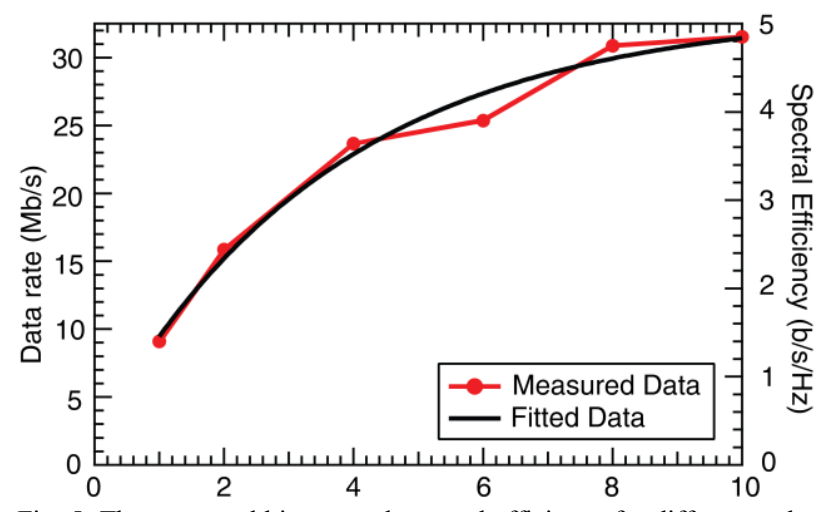

Fig. 5: The measured bit rate and spectral efficiency for different order of $m$-CAP with exponential fitting [18].

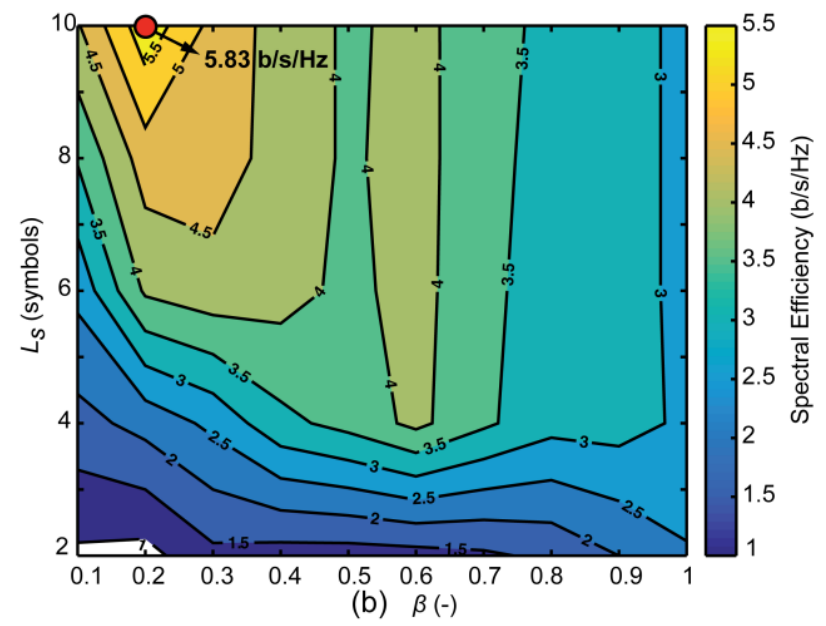

Fig. 6: Experimentally measured spectral efficiencies for 10-CAP for a range of $\beta$ and $L_{s}$ [31].

more accurate flat-band response approximation, and hence, higher SNR was measured for individual sub-carriers with improved data rates. However, the computational complexity was increased accordingly, recalling that $4 m$ filters are required, overall.

In [30], the experimental demonstration of $4 \times 4$ multipleinput multiple-output (MIMO) VLC system based on $m$-CAP was reported for a range of $m$, link distances and $B_{t o t}$ showing the trade-off between the measured data rate and spectral efficiency. By combining these two schemes (i.e., space and frequency multiplexing), the overall data rate of $\sim 249 \mathrm{Mb} / \mathrm{s}$ was measured for $m=20$ and $B_{t o t}$ of $20 \mathrm{MHz}$ at a link distance of $1 \mathrm{~m}$ and a BER of $10^{-3}$. The improvement in the spectral efficiency by up to $5.81 \mathrm{~b} / \mathrm{s} / \mathrm{Hz}$ per a channel for $m=10$ and $B_{t o t}=5 \mathrm{MHz}$ was achieved using the same transmission span of $1 \mathrm{~m}$ compared to the previous experiment reported in [18], which is a significant improvement, but with a further increase in computational complexity rising with the number of parallel channels.

Based on the results published in [21], the $m$-CAP VLC performance was experimentally investigated utilizing different filter parameters (i.e., $\beta$ and filter length $L_{s}$ ) as in [31]. The report showed that lower order systems can offer the same system performance as higher orders but, crucially, at much lower computational complexities. On the contrary, higher 
order $m$-CAP (i.e., $m \geq 6$ ) can be designed with the same filter parameters (i.e., $\beta$ and $L_{s}$ ) to support both the highest transmission speed and spectral efficiency, simultaneously. In comparison to [30], the spectral efficiency was slightly increased by up to $5.94 \mathrm{~b} / \mathrm{s} / \mathrm{Hz}$ for 8 -CAP at a $1 \mathrm{~m}$ distance. The example of measured spectral efficiencies of the 10-CAP system for a range of $\beta$ and $L_{s}$ is illustrated in Fig. 6 with the highlighted highest spectral efficiency measured in 10-CAP.

\section{CONCLUSION AND FUTURE OUTLOOK}

In this paper, we presented an overview on $m$-CAP modulation adopted in a VLC system. The concept of multiband approach of classical CAP scheme was adopted from optical fiber communications and a number of research works have been published showing the huge potentials of $m$-CAP. It was reported that $m$-CAP outperforms the single CAP system in terms of both the data rate and spectral efficiency. Moreover, the predicted values of spectral efficiency can reach up to 10 $\mathrm{b} / \mathrm{s} / \mathrm{Hz}$ for $m=20$. The highest experimentally demonstrated spectral efficiency was $5.94 \mathrm{~b} / \mathrm{s} / \mathrm{Hz}$ for 8 -CAP at a transmission span of $1 \mathrm{~m}$, which is significant and still provides enough headroom for further improvement compared to the theoretically predicted values. The majority of the system complexity is introduced by the pulse shaping filters, hence a new concept of $m$-CAP where the entire LED modulation bandwidth was allocated to the $1^{\text {st }}$ sub-carrier, was introduced in this work. Such a scheme reduced the number of FIR filters by $80 \%, 75 \%, 67 \%$ and $50 \%$ for $m=10,8,6$, and 4 , respectively, and even outperforms the traditional $m$-CAP in terms of the data rate by up to $36 \%$ for $m=6$.

The presented results have built a strong base for future research, which will be focused among others on the further reduction in the computational complexity, as well as increasing both the transmission speed and spectral efficiency with the emphasis on the experimental campaigns.

\section{ACKNOWLEDGEMENTS}

This work was supported by GACR 17-17538S project and the UK EPSRC grant EP/P006280/1: Multifunctional Polymer Light-Emitting Diodes with Visible Light Communications (MARVEL).

\section{REFERENCES}

[1] Cisco Visual Networking Index: Global Mobile Data Traffic Forecast Update, 2016-2021 White Paper, 2017.

[2] Z. Ghassemlooy, L. N. Alves, S. Zvanovec, and M. A. Khalighi, Visible Light Communications Theory and Applications. CRC Press, Boca Raton, 2017.

[3] Uysal, M., Capsoni, C., Ghassemlooy, Z., Boucouvalas, A. C., and Udvary E. G. (Eds.): Optical Wireless Communications - An Emerging Technology, Springer, 2016.

[4] Marchetti, N., "Towards $5^{\text {th }}$ generation wireless communication systems," in ZTE Communications, vol. 13, no. 1, pp. 11-19, 2015.

[5] A. Kumar, and M. Gupta, "Key Technologies and Problem in Deployment of 5G Mobile Communication Systems", in Communications on Applied Electronics, vol. 1, no. 3, pp. 4-7, 2015.

[6] Z. Bojkovic, and D. Milovanovic, "A Technology Vision of the Fifth Generation (5G) Wireless Mobile Networks," Proceedings of the First International Conference on Electrical, Electronic and Communications Engineering (ELECOM 2016), Bagatelle, Mauritius, pp. 25-43, 2016.
[7] J. Grubor, S. C. J. Lee, K. D. Langer, T. Koonen, and J. W. Walewski, "Wireless High-Speed Data Transmission with Phosphorescent WhiteLight LEDs," 33rd European Conference and Exhibition of Optical Communication - Post-Deadline Papers (published 2008), Berlin, Germany, 2007, pp. 1-2.

[8] X. Li et al., "Wireless visible light communications employing feedforward pre-equalization and PAM-4 modulation," in Journal of Lightwave Technology, vol. 34, no. 8, pp. 2049-2055, 2016.

[9] S. Rajbhandari, "Spatial and wavelength division multiplexing for highspeed VLC systems: An overview," 2016 10th International Symposium on Communication Systems, Networks and Digital Signal Processing (CSNDSP), Prague, 2016, pp. 1-6.

[10] J. F. Li, Z. T. Huang, R. Q. Zhang, F. X. Zeng, M. Jiang, and Y. F. Ji, "Superposed pulse amplitude modulation for visible light communication," in Optics Express, vol. 21, pp. 31006-31011, 2013.

[11] S. D. Dissanayake and J. Armstrong, "Comparison of ACO-OFDM, DCO-OFDM and ADO-OFDM in IM/DD systems," in Journal of Lightwave Technology, vol. 31, no. 7, pp. 1063-1072, 2013.

[12] G. Cossu, W. Ali, R. Corsini, and E. Ciaramella, "Gigabit-class optical wireless communication system at indoor distances $(1.5-4 \mathrm{~m})$, in Optics Express, vol. 23, pp. 15700-15705, 2015.

[13] H. Elgala, R. Mesleh, and H. Haas, "Nonlinearity effects and predistortion in optical OFDM wireless transmission using LEDs," in International Journal of Ultra Wideband Communications and Systems, vol. 1, no. 2., pp. 143-150.

[14] R. Mesleh, H. Elgala, and H. Haas, "LED nonlinearity mitigation techniques in optical wireless OFDM communication systems," in IEEE/OSA Journal of Optical Communications and Networking, vol. 4, no. 11 , pp. 865-875, 2012.

[15] F. M. Wu, C. T. Lin, C. C. Wei, C. W. Chen, H. T. Huang, and S. Chi, "Performance comparison of OFDM signal and CAP signal over high capacity RGB-LED-based WDM visible light communication," in IEEE Photonics Journal, vol. 5, no. 4, 2013.

[16] M. I. Olmedo, T. Zuo, J. B. Jensen, Q. Zhong, X. Xu, S. Popov, and I. T. Monroy, "Multiband carrierless amplitude phase modulation for high capacity optical data links," in Journal of Lightwave Technology, vol. 32, no. 4, pp. 798-804, 2014.

[17] J. Zhang, J. Yu, F. Li, N. Chi, Ze Dong, and X. Li, "11 × $5 \times 9.3 \mathrm{~Gb} / \mathrm{s}$ WDM-CAP-PON based on optical single-side band multi-level multiband carrier-less amplitude and phase modulation with direct detection," in Optics Express, vol. 21, pp. 18842-18848, 2013.

[18] P. A. Haigh, A. Burton, K. Werfli, H. Le Minh, E. Bentley, P. Chvojka, W. O. Popoola, I. Papakonstantinou, and S. Zvanovec, "A multi-CAP visible-light communications system with $4.85-\mathrm{b} / \mathrm{s} / \mathrm{Hz}$ spectral efficiency," in IEEE Journal on Selected Areas in Communications, vol. 33, no. 9, pp. 1771-1779, 2015.

[19] P. A. Haigh, A. Aguardo, Z. Ghassemlooy, P. Chvojka, K. Werfli, S. Zvanovec, E. Ertunç, and T. Kanesan, "Multi-band carrier-less amplitude and phase modulation for highly bandlimited visible light communications - Invited paper," 2015 International Conference on Wireless Communications \& Signal Processing (WCSP), Nanjing, 2015, pp. $1-5$.

[20] P. A. Haigh et al., "Multi-band carrier-less amplitude and phase modulation for bandlimited visible light communications systems," in IEEE Wireless Communications Magazine, vol. 22, no. 2, pp. 46-53, 2015.

[21] P. Chvojka, P. A. Haigh, S. Zvanovec, P. Pesek, Z. Ghassemlooy, "Evaluation of multi-band carrier-less amplitude and phase modulation performance for VLC under various pulse shaping filter parameters", Proceedings of the 13th International Joint Conference on e-Business and Telecommunications - Volume 3: OPTICS, (ICETE 2016).

[22] J. Wei, Q. Cheng, D. G. Cunningham, R. V. Penty, and I. H. White, "100$\mathrm{Gb} / \mathrm{s}$ hybrid multiband CAP/QAM signal transmission over a single wavelength," in Journal of Lightwave Technology, vol. 33, no. 2, pp. 415423, 2015.

[23] A. Tatarczak, M. Iglesias Olmedo, T. Zuo, J. Estaran, J. B. Jensen., X. Xu, and I. T. Monroy, "Enabling 4-lane based $400 \mathrm{G}$ client-side transmission links with multiCAP modulation," in Advances in Optical Technologies, vol. 2015, 2015.

[24] L. Sun, J. Du, and Z. He, "Multiband three-dimensional carrierless amplitude phase modulation for short reach optical communications," in Journal of Lightwave Technology, vol. 34, no. 13, pp. 3103-3109, 2016.

[25] K. Werfli, P. A. Haigh, Z. Ghassemlooy, P. Chvojka, S. Zvanovec, S. Rajbhandari, and S. Long, "Multi-band carrier-less amplitude and phase modulation with decision feedback equalization for bandlimited VLC 
systems," 2015 4th International Workshop on Optical Wireless Communications (IWOW), Istanbul, 2015, pp. 6-10.

[26] K. Werfli, P. A. Haigh, Z. Ghassemlooy, N. B. Hassan, and S. Zvanovec, "A new concept of multi-band carrier-less amplitude and phase modulation for bandlimited visible light communications," 2016 10th International Symposium on Communication Systems, Networks and Digital Signal Processing (CSNDSP), Prague, 2016, pp. 1-5.

[27] P. Chvojka, S. Zvanovec, K. Werfli, P. A. Haigh, and Z. Ghassemlooy, "Variable m-CAP for bandlimited visible light communications," 2017 IEEE International Conference on Communications Workshops (ICC Workshops), Paris, France, 2017, pp. 1-5.

[28] Y. Wang, L. Tao, Y. Wang, and N. Chi, "High speed WDM VLC system based on multi-band CAP64 with weighted pre-equalization and modified CMMA based post-equalization," in IEEE Communications Letters, vol. 18, no. 10, pp. 1719-1722, 2014.

[29] P. A. Haigh et al., "Experimental verification of visible light communications based on multi-band CAP modulation," 2015 Optical Fiber Communications Conference and Exhibition (OFC), Los Angeles, CA, 2015, pp. 1-3.

[30] K. Werfli, P. Chvojka, Z. Ghassemlooy, N. B. Hassan, S. Zvanovec, A. Burton, and P. A. Haigh, "Experimental demonstration of high-speed $4 \times$ 4 imaging MIMO visible light communications employing multi-CAP," in Journal of Lightwave Technology, submitted.

[31] P. Chvojka, K. Werfli, S. Zvanovec, P. A. Haigh, V. Hubata Vacek, P. Dvorak, P. Pesek, and Z. Ghassemlooy, "On the m-CAP performance with different pulse shaping filters parameters for visible light communications," in IEEE Photonics Journal, submitted. 